\title{
Caracterización de la resistencia de Amaranthus quitensis a tres familias de herbicidas
}

\author{
María V. Romagnoli ${ }^{\varpi}$; Daniel Tuesca \& Hugo R. Permingeat \\ Facultad de Ciencias Agrarias. Universidad Nacional de Rosario, Santa Fe, Argentina
}

\begin{abstract}
Resumen. Amaranthus quitensis es una especie anual de origen sudamericano que se reproduce por semillas. En Argentina su distribución abarca centro y norte del país y constituye una de las principales malezas en los cultivos de verano (soja, maíz y girasol) del área pampeana. En la región central (provincia de Córdoba y Santa Fe) se detectaron fallas en su control al emplearse distintas familias de herbicidas (imidazolinonas, sulfonilureas y triazolopirimidinas) por lo cual el objetivo de este trabajo fue caracterizar la resistencia a nivel bioquímico y molecular de biotipos de A. quitensis correspondientes a cuatro localidades de esta región. Específicamente utilizamos distintos herbicidas inhibidores de la Aceto-Lactato Sintasa (ALS), una enzima involucrada en la síntesis de un grupo de aminoácidos, tomando como base de comparación un biotipo susceptible. Como objetivos específicos determinamos el origen geográfico de la resistencia a cada uno de estas tres familias de herbicidas y evaluamos el efecto de diferentes dosis sobre la actividad de la ALS "in vitro". Los resultados indican que los materiales analizados corresponden a eventos de resistencia independientes y no a un origen común por movilidad de germoplasma entre las localidades en donde se detectaron las resistencias. Los estudios de variabilidad genética de los biotipos estudiados aportan evidencias de los mecanismos de resistencia frente a diferentes herbicidas que tienen como blanco la enzima ALS.
\end{abstract}

[Palabras clave: ALS, resistencia a acetolactato sintasa, biodiversidad, agroecosistemas]

\begin{abstract}
Aвstract. Characterization of Amaranthus quitensis resistance to three families of herbicides: Amaranthus quitensis is an annual species from South America that reproduces by seed. In Argentina its distribution covers central and northern country and is a major weed of summer crops (soybean, corn and sunflower) Pampa area. In the central region (Province of Cordoba and Santa Fe) flaws in its control were detected, when using different herbicide families (imidazolinone, sulfonylurea and triazolopyrimidines). Therefore, the aim of this study was to characterize the resistance at the biochemical and molecular level of biotypes of $A$. quitensis from four localities along this region. Specifically we used different herbicides inhibitors Aceto-Lactate Synthase (ALS), an enzyme involved in the synthesis of a group of aminoacids, and compared them with a susceptible biotype. Specific objectives were to determine the geographical origin of the resistance to each of these three herbicides and to assess the effect of different doses on ALS activity in vitro. Results, based on the observed genetic variability and on the sequences of the als genes, indicated that the analyzed populations were originated independently and not from a common mutation event that later dispersed to the different sites. These results allowed for a deeper knowledge at the biochemical and molecular levels of the resistance mechanisms of five ecotypes of A. quitensis to different herbicides that have the ALS enzyme as a target.
\end{abstract}

[Keywords: ALS, acetolactate synthase resistance, biodiversity, agroecosystems]

\section{INTRODUCCIÓN}

Amaranthus quitensis es una especie anual de origen sudamericano que se reproduce por semillas. Pertenece a la familia de las Amarantáceas, que comprende más de 60 especies entre las que se encuentran algunas comestibles, difundidas en las regiones cálidas y templadas (Burkart 1978). En nuestro país, esta maleza abarca todo el territorio, con excepción del área comprendida al sur de la provincia de Río Negro. En la región pampeana es una de las principales malezas en los cultivos de verano (soja, maíz y girasol). Esta especie es susceptible a muchos de los herbicidas recomendados para el control de especies dicotiledóneas en los sistemas agrícolas.

\valeria@express.com.ar
El uso de herbicidas de acción selectiva supone que determinadas especies cultivadas (y no cultivadas) son capaces de desarrollarse a las dosis recomendadas de aplicación agrícola de esos herbicidas, aunque puedan ser controladas a dosis superiores. Un grupo interesante de herbicidas son los que corresponden a los inhibidores de la enzima Aceto-Lactato Sintasa (ALS), involucrada en la síntesis de algunos aminoácidos, debido a las bajas dosis de uso, baja toxicidad y bajo riesgo ambiental. Sin embargo, las bases de datos de especies que generaron resistencia a estos herbicidas informan la existencia de 116 especies (Heap 2012). El primer herbicida inhibidor de la ALS comercializado fue el clorsulfurón en 1982 y solo 5 años después

\section{Editora Asociada: Marina Omacini} 2013; Revisión recibida: 3 de mayo; Aceptado: 7 de mayo. 
se confirmó la aparición de resistencia en una población de Lactuca serriola (Mallory Smith et al. 1990). La rápida aparición de casos de resistencia cataloga a estos grupos como de alto riesgo para la evolución de resistencia.

De acuerdo con lo analizado por Cristoffoleti et al. (2000), en cualquier población donde los individuos muestren una base genética variable en cuanto a tolerancia a una medida de control, la aplicación en forma repetida de esta medida puede cambiar la composición poblacional, promoviendo el éxito diferencial de los individuos resistentes y disminuyendo la eficacia de esta estrategia. En las poblaciones de malezas, independientemente de la aplicación de cualquier herbicida, existen individuos con características genéticas que le confieren resistencia a ese principio activo.

Para comprender cómo se genera la resistencia a herbicidas en una población de malezas se han propuesto dos mecanismos: la teoría de la mutación (cambio genético) o cambios en la población de la maleza por genes preexistentes que confieren resistencia a la población (selección natural) (Kissmann 2003). En el primer caso, la resistencia puede acontecer por la ocurrencia de mutaciones genéticas que dotan a la población de resistencia al herbicida. Estas mutaciones ocurren en frecuencias muy bajas, pueden ocurrir independientemente de la aplicación del herbicida y no existen evidencias de que sean inducidas por la acción del herbicida (Duke et al. 1991). Los principales factores que afectan la evolución de la resistencia en plantas han sido agrupados en genéticos, bioecológicos y agronómicos. Los factores genéticos son inherentes a los individuos de una misma población de malezas. Los bioecológicos comprenden una interacción entre las características de los individuos y la acción del ecosistema sobre esa población y los agronómicos son la resultante de la selección inducida por las prácticas agrícolas. Los dos primeros son de difícil manipulación para el manejo de la resistencia mientras que los agronómicos dependen de manera directa de la acción antrópica para implementar estrategias de manejo (Matiello et al. 1999).

La base genética de la resistencia, su herencia y la forma de reproducción de las especies son aspectos relevantes en su evolución y dispersión. El análisis de estos aspectos es requisito para la conceptualización racional de pautas de mitigación. La resistencia a inhibidores de la ALS suele involucrar genes dominantes o con dominancia incompleta. La resistencia conferida por un gen dominante de origen nuclear se propaga rápidamente en las especies de fecundación cruzada; la dispersión del polen contribuirá de forma considerable al flujo genético de la resistencia en la población y a la dispersión de la resistencia hacia otras poblaciones.

El mecanismo de acción de estos herbicidas consiste en una inhibición de la ALS, que conduce a la muerte prematura de la planta, principalmente como resultado de la falta de aminoácidos de cadena lateral ramificada (Shaner et al. 1991). Si bien estos compuestos no compiten por el sitio de unión al sustrato o a cofactores, Scholss et al. (1988) demostraron que sulfonilureas, imidazolinonas y triazolopirimidinas sí compiten por la unión a la ALS (Hawkes 1989; Subramanian \& Gerwick 1989). Se determinó que las mutaciones que confieren resistencia a inhibidores de la ALS en general constituyen el alelo dominante y reducen la unión de algunas o todas las clases de inhibidores de la ALS a la enzima, (Tranel \& Wright 2002). La mayoría de los casos de resistencia a inhibidores de la ALS resultaron de la selección de un sitio blanco alterado, generado por una o varias mutaciones puntuales en la enzima. Por ejemplo, la sustitución de la $\mathrm{Ala}_{122}$ por Thr es la base de una resistencia natural IM-específica en una población de $A$. hybridus (Trucco et al. 2006). Esta mutación puntual también ha sido identificada como la base de la resistencia a inhibidores de la ALS en otras especies de malezas como Xanthium strumarium (Bernasconi et al. 1995), Solanum ptycanthum (Milliman et al. 2000) y A. retroflexus (Mc Naughthon et al. 2005). Otra mutación identificada en la ALS que confiere resistencia a herbicidas en $A$. hybridus fue la sustitución del $\operatorname{Trp}_{574}$ por Leu (Maertens et al. 2004). La resistencia a sulfonilureas e imidazolinonas en plantas está asociada a mutaciones puntuales que provocan sustituciones de aminoácidos para los residuos Ala ${ }_{122}$, Pro $_{197}, \operatorname{Trp}_{574}$ o Ser (Tranel \& Wright 2002). Yu et al. (2010) encontraron que diferentes mutaciones de la ALS en poblaciones resistentes de Lolium rigidum condujeron a una mayor actividad de la enzima, sin modificar de manera importante su cinética y el crecimiento de las plantas. También se encontró que diferentes mutaciones en el gen codificante de la ALS de plantas resistentes de Raphanus raphanistrum no imponen efectos pleiotrópicos negativos sobre su tasa de crecimiento, fotosíntesis y habilidad competitiva (Li et al. 2012). 
Tuesca \& Nisensohn (2001) identificaron cuatrobiotipos de A.quitensis quesobrevivieron a la aplicación de herbicidas inhibidores de la ALS en condiciones de campo y caracterizaron los niveles de resistencia mediante ensayos de dosis-respuesta. El objetivo principal de nuestro trabajo fue caracterizar, en esos cuatro biotipos, la resistencia a nivel bioquímico y molecular frente a distintos herbicidas inhibidores de la ALS. Además, para los biotipos que comparten resistencia a la misma familia de herbicidas, se analizó su grado de parentesco, con el fin de evaluar el papel de la dispersión de los ecotipos resistentes como consecuencia del sistema productivo agrícola.

\section{MATERIALES Y MÉTODOS}

Se cosecharon semillas de plantas de A. quitensis en campos en los que se detectaron fallas en el control de esta maleza al emplearse herbicidas de las familias de las sulfonilureas, imidazolinonas y triazolopirimidinas. Asimismo se recolectaron semillas de la población Zavalla, en la cual no se observaron deficiencias en el control químico (Tuesca \& Nisensohn 2001), por lo que se tomó como control susceptible. Los materiales evaluados llevan el nombre de la localidad de la cual provienen: General Baldissera $\left(33^{\circ} 07^{\prime}\right.$ Sur y $\left.62^{\circ} 19^{\prime} \mathrm{O}\right)$; Las Rosas ( $32^{\circ} 29^{\prime} \mathrm{S}$ y $\left.61^{\circ} 35^{\prime} \mathrm{O}\right)$; Pueblo Italiano (335' 5 y $62^{\circ} 50^{\prime} \mathrm{O}$ ); Marcos Juárez ( $32^{\circ} 41^{\prime}$ S y $\left.62^{\circ} 06^{\prime} \mathrm{O}\right)$ y Zavalla $\left(33^{\circ} 0^{\prime} \mathrm{S}\right.$ y $\left.60^{\circ} 53^{\prime} \mathrm{O}\right)$.

\section{Ensayos de resistencia in vivo}

Para la obtención del material experimental se sembraron 60 semillas de los cuatro biotipos resistentes y del biotipo susceptible en recipientes plásticos conteniendo una mezcla de tierra, arena y perlita (70-20-10\%, respectivamente) esterilizada previamente con bromuro de metilo. Al momento en que las plantas poseían de 4 a 6 hojas verdaderas se aplicaron las siguientes dosis de herbicidas: $1000 \mathrm{~g} \mathrm{pa} /$ ha de Imazetapir (imidazolinonas); 100 g pa/ha de Clorimurón-etil (sulfonilureas) y 1200 g pa/ha de Flumetsulam (triazolopirimidinas). Estas concentraciones representan 10 veces la dosis de uso recomendada en el marbete de las respectivas formulaciones comerciales. De cada biotipo se mantuvieron testigos a los cuales no se les aplicó ningún tratamiento químico. Los herbicidas fueron aplicados utilizando una mochila de presión constante con fuente de $\mathrm{CO}_{2}$ equipada con pastillas XR11001. La presión fue de $241 \mathrm{kPa}$ y el caudal de $130 \mathrm{~L} / \mathrm{ha}$. A tres plantas sobrevivientes de los tratamientos herbicidas de cada biotipo se les cubrió las inflorescencias de manera de evitar la polinización cruzada con individuos cercanos. Las panojas de cada planta se cosecharon por separado al finalizar el ciclo. Se germinó una muestra de semillas de cada planta para obtener la generación filial (alrededor de 30 individuos), la cual fue utilizada para los ensayos de resistencia "in vitro".

\section{Ensayos de resistencia in vitro}

Se extrajeron proteínas de hojas de plantas jóvenes (21 días de germinadas) tanto de los biotipos resistentes como del control de acuerdo al método propuesto por Poston et al. (2002) y la actividad enzimática se determinó midiendo la cantidad de acetoína formada en función de las dosis ensayadas de los diferentes herbicidas, según el método de Westerfeld (1945). En este método se desarrolla un color rosa en un medio que además contiene creatina y $\alpha$-naftol. La intensidad de este color se cuantificó midiendo absorbancia a $530 \mathrm{~nm}$. Las dosis de herbicidas utilizadas para los ensayos de cinética enzimática fueron: $0 ; 0.05 ; 0.5 ; 5$ y $50 \mu \mathrm{M}$ para imidazolinonas; $0 ; 0.05 ; 0.5 ; 5$ y $25 \mu \mathrm{M}$ para sulfonilureas y $0 ; 10 ; 100 ; 500$ y $1000 \mu \mathrm{M}$ para triazolopirimidinas. Los valores de inhibición obtenidos se interpretaron considerando un valor de $\mathrm{I}_{\mathrm{p}}$ que representa la concentración de herbicida requerida para reducir la actividad enzimática en un porcentaje " $p$ ", comparado con el testigo sin tratar.

\section{Extracción de ADN y análisis de diversidad genética de los biotipos}

Se extrajo ADN genómico de hojas turgentes provenientes de individuos de todos los biotipos (resistentes y susceptible) de acuerdo al método informado por Lipp et al. (1999). La diversidad genética de los biotipos resistentes y del control se determinó mediante análisis de fragmentos de ADN polimórficos amplificados al azar (RAPDs, por sus siglas en inglés) (Williams et al. 1990), utilizando 20 cebadores provistos por la UBC (University of British Columbia), Canada, series 300-399. Los datos de presencia/ausencia de cada banda de amplificación fueron utilizados para generar una matriz de similitud genética de acuerdo al índice de Jaccard y un fenograma.

Las reacciones de amplificación se realizaron utilizando la metodología descripta en el Manual de Laboratorio del CIMMYT, 2006. Las condiciones de amplificación de fragmentos de ADN por reacción en cadena de la polimerasa (PCR) fueron las siguientes: buffer Gotaq green (Promega) 1X $200 \mu \mathrm{M}$ dNTPs, $0.3 \mu \mathrm{M}$ oligonucleótidos, $1.25 \mathrm{U} /$ tubo Taq (Promega), $100 \mathrm{ng}$ de ADN (2 $\mu \mathrm{L})$. La amplificación consistió en una etapa de desnaturalización a $95{ }^{\circ} \mathrm{C}$ durante 1 minuto, 46 ciclos de $95^{\circ} \mathrm{C}$ durante 1 minuto, $36^{\circ} \mathrm{C}$ por 1 minuto y $72^{\circ} \mathrm{C}$ por 1 minuto, y una etapa final de extensión a $72{ }^{\circ} \mathrm{C}$ durante 5 minutos. Los amplicones obtenidos en las PCRs fueron resueltos en geles de agarosa al 2-2.5\%, y sometidos a electroforesis a $60 \mathrm{~mA}$ en TAE 1X. Luego, los geles se tiñeron incubándolos 10 minutos con una solución de $1 \mu \mathrm{g} / \mathrm{mL}$ bromuro de etidio y se observaron en transiluminador UV (254nm). 


\section{RESUltadOS Y DisCUSIÓN}

\section{Ensayos de resistencia in vivo}

Las aplicaciones de los herbicidas, en una dosis 10 veces mayor a la recomendada en el marbete, confirmaron la resistencia de los biotipos a los respectivos ingredientes activos. El efecto se registró a los 15 días de la aplicación, momento en que las plantas poseían 4-6 hojas verdaderas. El biotipo susceptible evidenció una mortalidad del $100 \%$ con todos los herbicidas aplicados. Los biotipos Las Rosas y Marcos Juárez también resultaron muy susceptibles en los tratamientos con sulfonilureas y triazolopirimidinas, presentando también una mortalidad del 100\%. Frente al tratamiento con imidazolinonas, Marcos Juárez mostró una leve disminución de crecimiento y desarrollo, comportándose como resistente a este principio activo. Por su parte, los biotipos Baldissera y Pueblo Italiano tratados con los diferentes principios activos sólo mostraron una disminución del crecimiento en comparación al testigo sin tratar y mantuvieron una coloración verde intensa, terminando su ciclo de vida de igual manera que el testigo. Estos ensayos demuestran diferencias de respuesta en los niveles de resistencia de los biotipos al tratamiento con herbicidas inhibidores de la ALS.

\section{Ensayos de resistencia in vitro}

La actividad enzimática de la ALS confirmó las diferencias de resistencia existentes entre los biotipos, los principios activos y las dosis ensayadas (Figura 1). En ella se representa la actividad ALS como porcentaje sobre el testigo $(100 \%)$, relacionándola con el Log de la dosis de herbicida empleada. Cuando se utilizaron imidazolinonas (Figura 1a), la cinética de inhibición difirió entre los extractos proteicos de los distintos biotipos. La actividad ALS fue inhibida a la mitad de su nivel original $\left(\mathrm{I}_{50}\right)$ con una dosis de $0.05 \mu \mathrm{M}$ para el biotipo Las Rosas, mientras que esta dosis inhibió la actividad de la enzima en un 35\% al biotipo susceptible. Para alcanzar este mismo nivel de inhibición de la actividad de la enzima en el resto de los biotipos se requirió un rango de dosis de $5 \mu \mathrm{M}$ a más de $50 \mu \mathrm{M}$ en el siguiente orden para los restantes biotipos: Marcos Juárez $<$ Pueblo Italiano $<$ Baldissera. Cuando se utilizaron sulfonilureas, la respuesta fue muy diferente. En la Figura 1b sólo se representa el comportamiento del biotipo utilizado como control susceptible (Zavalla) comparado con la respuesta del biotipo Baldissera, ya que la actividad de los biotipos restantes fue similar a la presentada por el susceptible para dosis del herbicida de $0.05 \mu \mathrm{M}$. Los resultados indican que fue necesaria una dosis de $0.5 \mu \mathrm{M}$ para alcanzar la $\mathrm{I}_{50}$ en el biotipo Baldissera, siendo el único resistente a este principio activo.

La información que surge de este ensayo es un claro ejemplo de la elevada presión de selección que se aplica con este tipo de herbicidas, ya que utilizando dosis significativamente inferiores a las recomendadas se lograría controlar en forma eficiente cuatro de los cinco biotipos evaluados. Cuando se utilizan triazolopirimidinas (Figura 1c), los biotipos
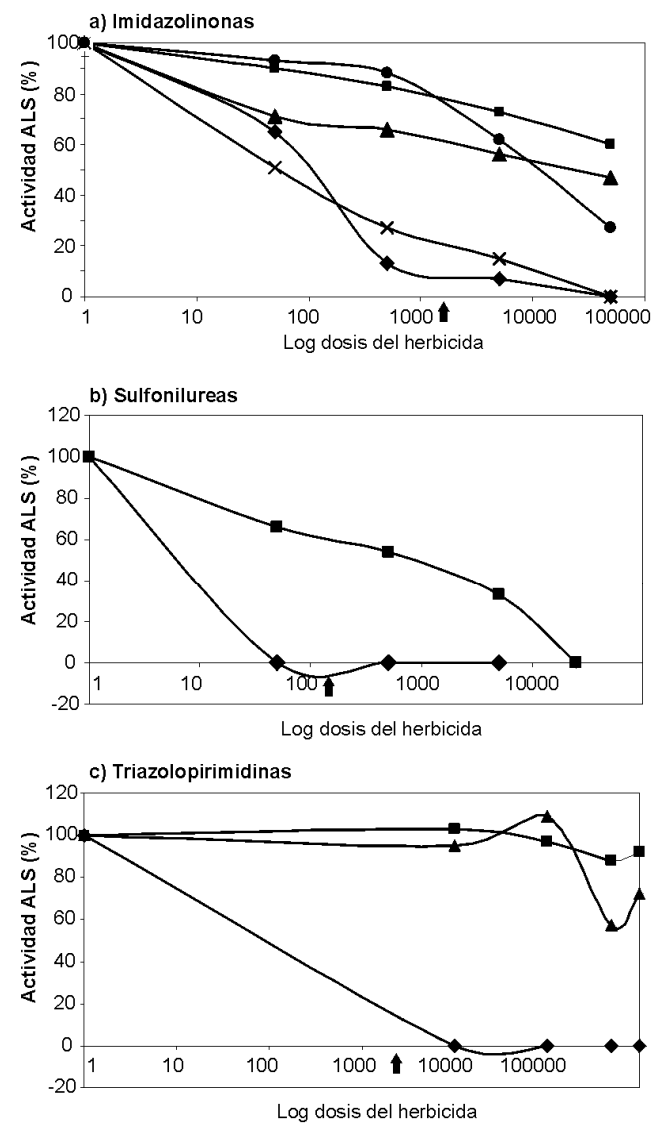

$\rightarrow$ Zavalla $\rightarrow$-Baldissera $\longrightarrow$ Pueblo Italiano $\rightarrow$-Las Rosas $\rightarrow$ Marcos Juarez

Figura 1. Actividad ALS relativa (\%) de los biotipos estudiados en función del Log de la dosis empleada para los tres herbicidas utilizados. Los puntos corresponden a la media de tres mediciones. La flecha indica la dosis de campo. Familias de herbicidas: a) Imidazolinonas, b) Sulfonilureas, y c) Triazolopirimidinas. Los biotipos no representados en las gráficas b y c mostraron una respuesta similar al control susceptible $(\mathrm{Za})$.

Figure 1. Relative ALS activity (\%) of the biotypes studied, according to the Log of the applied dose for the three used herbicides. Points correspond to the mean of three meassurments. The arrow indicates the field dose. Herbicides families: a) Imidazolinone, b) Sulfonylurea, and c) Triazolopyrimidines. Biotypes absents in graphs $\mathrm{b}$ and $\mathrm{c}$ showed a similar response to the susceptible control (Za). 
Baldissera y Pueblo Italiano mostraron una respuesta semejante, esto es una mayor actividad ALS respecto del testigo en la mayoría de las dosis ensayadas. Con una dosis de $1000 \mu \mathrm{M}$, que representa 500X la dosis de campo, se observaron valores de $91.6 \%$ y $72.0 \%$ de actividad para Ba y PI, respectivamente. Esto indica que con muy altas dosis de este herbicida, la actividad ALS continúa siendo suficientemente elevada como para permitir la superviviencia de la planta. Los restantes biotipos mostraron un comportamiento similar al biotipo susceptible (datos no mostrados).

Los experimentos de actividad enzimática con los herbicidas de las familias de las imidazolinonas y sulfonilureas se realizaron por triplicado mientras que con las triazolopirimidinas se hicieron por sextuplicado para confirmar la observación que no existía una disminución de la actividad en función de la dosis. La respuesta fue consistente en todos los casos y para las triazolopirimidinas podríamos hablar de una insensibilidad de la enzima a este principio activo en los biotipos Baldissera y Pueblo Italiano. Estos resultados estarían indicando que la resistencia al herbicida observada en los biotipos estudiados es del tipo de sitio de acción siendo específica para las triazolopirimidinas. Los mecanismos adicionales de resistencia (metabolismo de detoxificación, reducción, captación y translocación) se presumen no significativos en función de las observaciones y por la elevada magnitud de la insensibilidad a la inhibición de la enzima (500X) (Trucco et al. 2006).

\section{Diversidad genética de los biotipos}

La resistencia a herbicidas es un ejemplo de la evolución como consecuencia de los cambios causados en el ambiente por las prácticas agropecuarias (Maxwell \& Mortimer 1994). Los mecanismos de dispersión de semillas o polen pueden clasificarse en abióticos (por acción del viento y el agua) y bióticos (por el transporte externo del material adherido al pelaje, miembros, plumas, etc., a través de la ingesta y de la intervención de insectos). Por su parte, la actividad del hombre afecta tanto a factores abióticos como bióticos (Benvenuti 2007). Estos mecanismos de dispersión pueden influenciar el patrón de movimiento de los genes de la resistencia.

Para determinar si los biotipos analizados y resistentes a un mismo herbicida derivan de una mutación original que se dispersó en la región o, si por el contrario, la resistencia surgió de eventos independientes, se realizó una evaluación de las diferencias genéticas existente entre los biotipos por medio de análisis de RAPDs. Es importante tener en cuenta que en estos sistemas productivos es muy común el movimiento de las maquinarias agrícolas entre regiones, lo que estaría ayudando en la dispersión de las semillas. De los 20 cebadores ensayados, 15 resultaron informativos(amplificaronbandas), brindando un total de 33 fragmentos amplificados que se consideraron para el análisis.

En promedio, los biotipos mostraron valores de similitud de 0.45 (Figura 2). La máxima similitud entre los biotipos es de 0.61 (ZavallaLas Rosas) y la mínima es de 0.41 (Zavalla-Las Rosas vs. Marcos Juárez-PuebloJuárez-Pueblo Italiano). Asimismo, se observa la existencia de tres grupos de poblaciones, uno de los cuales está conformado por los biotipos Zavalla y Las Rosas con una similitud de 0.61, como se mencionara antes, estando el resto separado por similitudes de 0.57 (Pueblo Italiano y Marcos Juárez) y en forma aislada del resto

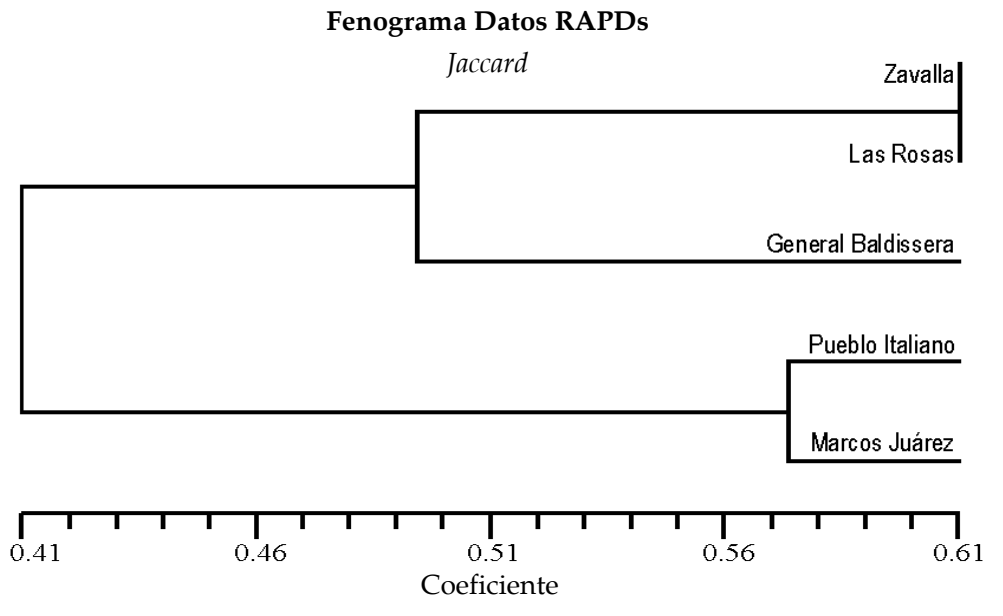

Figura 2. Análisis de agrupamiento UPGMA en base a los datos de similitud de Jaccard (1908).

Figure 2. UPGMA cluster analysis, according to Jaccard similarity data (1908). 
Baldissera con sólo un valor de 0.49 respecto al primero. Sobre la base de estos resultados, interpretamos que los diferentes biotipos corresponden a fondos genéticos distintos y no es posible identificar similitudes genéticas entre los biotipos asociadas a la resistencia (esto es, los más parecidos Zavalla y Las Rosas son susceptible y tolerante, respectivamente, para el caso de las imidazolinonas).

La localidad más cercana a Zavalla (susceptible) es Las Rosas $(86.7 \mathrm{~km})$ y en el fenograma estas localidades aparecen como el grupo de mayor similitud genética, aunque no son las localidades más cercanas en kilómetros de todas las analizadas. En contraposición, Marcos Juárez y Baldissera son las localidades más cercanas entre sí $(49.5$ $\mathrm{km}$ ), pero según el resultado obtenido en el fenograma pertenecen a grupos separados de similitud genética. Por último, la máxima distancia es de $193.3 \mathrm{~km}$ entre Las Rosas y Pueblo Italiano, una distancia muy probable de recorrer por las maquinarias en el momento de máxima actividad agrícola, pero sus fondos genéticos no son compartidos. Con este análisis podríamos corroborar los resultados obtenidos con la aplicación del coeficiente de Jaccard, afirmando que los diferentes biotipos corresponden a fondos genéticos distintos.

Es importante destacar que los cuatro biotipos resistentes provienen de sistemas donde la siembra directa de cultivos de verano y aplicaciones sistemáticas de herbicidas en altas dosis constituyen una práctica habitual desde hace casi 15 años. Por el contrario, el biotipo susceptible proviene de un lote donde se realiza un esquema de rotaciones de cultivos de verano, aplicando laboreo convencional y rotando en forma permanente los principios activos de herbicidas utilizados.

Según Benvenuti (2007), la forma de dispersión de las semillas de $A$. retroflexus podría considerarse barocórica (por gravedad), la cuál no sería un verdadero mecanismo de dispersión, sino que se utiliza en ausencia de un dispositivo para poder movilizar las semillas desde la planta madre. Las especies barocóricas son susceptibles de ser dispersadas por el hombre en la actividad agropecuaria. De acuerdo a nuestros resultados podríamos considerar que los eventos de resistencia evaluados no tuvieron su origen en una dispersión antropocórica, sino que habrían resultado de eventos independientes.

En síntesis, los resultados presentados en este trabajo indican que si bien los materiales analizados pueden haber tenido un origen común por movilidad de germoplasma entre las localidades de donde se detectaron las resistencias, los estudios de variabilidad genética indican que cada biotipo responde a un evento de resistencia independiente.

Agradecimientos: Agradecemos al Dr. J.P. Ortiz por la ayuda en el análisis bioinformático de los datos.

\section{BIBLIOGRAFÍA}

Benvenuti, S. 2007. Weed seed movement and dispersal strategies in the agricultural environment. Weed Biology and Management, 7:141-157.

BERNASCONI,P;ARWOODWORTH;BA ROSEN;MVSUBRAMANIAN \& DL SIEHL. 1995. A naturally occurring point mutation confers broad range tolerance to herbicides that target acetolactate synthase. J. Biol. Chem., 270:17381-17385.

Burkart, A. 1978. Flora ilustrada de Entre Ríos. Tomo VI, Parte 3. Editado por INTA. Pp. 160-173.

CRistoffoleti, PJ; D Medeiros; PA Monqueiro \& T PAssini. 2000. Planta Daninha á Cultura da Soja: Controle Químico e Resisténcia a Herbicidas. Soja: tecnología da produçao/ editado por Gil Miguel de Sousa Cámara. Piracicaba: ESALQ/pp. 179-202.

Duke, So; AL Christy; FD Hess \& ZS Holt. 1991. Herbicide Resistance Crops. Comments from CAST 1991-1. Council of Agricultural Science and Tecnology, Ames IA.

HAWKES, TR. 1989. Studies of herbicides which inhibit branched chain amino acid biosynthesis. En: Copping, LG; J Dalziel; Dodge AD (eds.). Prospects for Amino Acid Biosynthesis Inhibitors in Crop Protection and Pharmaceutical Chemistry. BCPC Monograph 42:131138.

HEAP, IM. The International Survey of Herbicide Resistant Weeds. Online. Internet. 2012. Disponible en www.weedscience.com.

KIssmanN, KG. Resistencia de plantas deninhas a herbicidas. Disponible en: www.hrac-br.com.br/ arquivos/texto_herbicidas.doc.01/05/2003a.

LI, M; Q Yu; H HaN; M VILA-Aiub; SB Powles. 2012. ALS herbicide resistancemutations in Raphanus raphanistrum: evaluation of pleiotropic effects on vegetative growth and ALS activity. (wileyonlinelibrary.com) DOI 10.1002/ pp. 3419.

Lipp, M; P Brodmann; K Pietsch; J Pauwels \& E AnKlam. 1999. IUPAC collaborative trial study of a method to detect genetically modified soybeans and maize in dried powder. J. AOAC Int., 82: 923-928.

Maertens, KD; CL Sprague; PJ Tranel \& RA Hines. 2004. Amaranthus hybridus populations resistant to triazine and acetolactate synthase-inhibiting herbicides. Weed Res., 44:21-26.

Mallory Smith, CA; DC Thill \& MJ Dial. 1990. Identification of sulfonylureas herbicide-resistant prickly lettuce (Lactuca serriola). Weed Technology, 4: 163-168.

Matiello, RR; JP Ronzelli \& C PuRíssimo. 1999. Mecanismos de resistencia: fatores biológicos, agronómicos e genéticos. Anais/2. Curso de manejoda resistencia de plantas daninhas aos herbicidas. Ponta Grossa, PR: AEACG. Pp. 27-40.

MAXWELL, MD \& M MORTIMER. 1994. Selection for herbicide 
resistance. Pp 1-25 en: Powles, SB \& JAM Holtum. Herbicide Resistance in Plants: Biology and Bioquemistry. (eds.). CRC press, Boca Ratón.

McNaughton, KE; J Letart \& EA Lee. 2005. Mutations in ALS confer herbicide resistance in redroot pigweed (Amaranthus retroflexus) and Powell amaranth (Amaranthus powellii). Weed Sci., 53:17-22.

Milliman, LD; DE Riechers; FW Simmons \& LM Wax. 2000. Two biotypes of eastern black nightshade that are resistant to ALS inhibiting herbicides. Proc. N. Cent. Weed Sci. Soc., 55:86.

Poston, HP; CM HiRATA \& HP WiLson. 2002. Response of acetolactate synthase from imidazolinone-susceptible and resistant smooth pigweed to ALS inhibitors. Weed Science, 50:306-311.

SCHLOSS, JV; LM CISKANIK \& DE VAN DYK. 1988. Hawkes Origin of the herbicide binding site of the acetolactate synthase. Nature, 331:360-362.

Schmitzer, PR; RJ EILER \& C CséKe. 1993. Lack of crossresistance of imazaquinresistant Xanthium strumarium acetolactate synthase to flumetsulam and chlorimuron. Plant Physiol., 103:281-283.

SHANER, DL. 1991. Physiological effects of theimidazolinone herbicides. En: Shaner, DL \& SL O'Connor (eds.). The imidazolinone herbicides Ann. Arbor. Lewi. Pp. 129-138.

Sibony, M; A Michel H; U Haas; B Rubin \& K Hurle. 2001. Sulfometuron-resistant Amaranthus retro exus: cross-resistance and molecular basis for resistance to acetolactate syntase-inhibing herbicides. Black well Science Ltd. Weed Research, 41:491-508.

Subramanian, MV \& BC Gerwick. 1989. Inhibition of acetolactate synthase by triazolopyrimidines: a review of recent develop- ments. Pp. 277-288 20 en: Whitaker, JR \& PE Sonnet (eds.). Biocatalysis in Agri- cultural Biotechnology. ACS Symposium Series No. 389. American Chemical Society, Washington, DC.

TRANeL, PJ \& TR WRight. 2002. Review: Resistance of weeds to ALS-inhibing herbicides: what heve we leRNAed? Weed Science, 50:700-712.

Trucco, F; AG Hager \& P Tranel. 2006. Acetolactate syntase mutation conferring imidazolinone-specific herbicide resistance in Amaranthus hybridus. Journal of Plant Physiology, 163:475-479

TuEsCA, D \& L NisensoHn. 2001. Resistencia de Amaranthus quitensis a imazetapir y clorimurón-etil. Pesq. Agrop. Bras., 36:601-606.

Westerfeld, WW. 1945. A colorimetric determination of blood acetoin. J. Biol. Chem., 161:495-502.

Williams, JKG; AR KUBELIK; KJ LiVAK; JA RAFALSKY \& SV TYNGER. 1990. DNA polymorphisms amplified by arbitrary primers are useful as genetic markers. Nucleic Acids Res., 18: 6531-6535

Yu, Q; H HaN; M VILA-AiUB \& SB Powles. 2010. AHAS herbicide resistance endowing mutations: effect on AHAS functionality and plant growth. Journal of Experimental Botany, 61:3925-3934. 